Journal of Animal and Veterinary Advances 11 (19): 3581-3587, 2012

ISSN: $1680-5593$

(C) Medwell Journals, 2012

\title{
Application of Autologous Mesenchymal Stem Cells and Coenzyme Q10 in a Pig Model of Acute Myocardial Infarction
}

\author{
${ }^{1}$ Sheng-Chuan Lin, ${ }^{4}$ Tzong-Fu Kuo, ${ }^{5} \mathrm{Fu}-J e n$ Huang, ${ }^{2}$ Tsung-Chou Chang and ${ }^{3}$ Yan-Der Hsuuw \\ ${ }^{1}$ Department of Tropical Agriculture and International Cooperation, \\ ${ }^{2}$ Department and Graduate Institute of Veterinary Medicine, School of Veterinary Medicine, \\ ${ }^{3}$ Department of Biological Science and Technology, \\ National Ping-Tung University of Science and Technology, 91201 Pingtung, Taiwan \\ ${ }^{4}$ Department and Graduate Institute of Veterinary Medicine, School of Veterinary Medicine, \\ National Taiwan University, 10617 Taipei, Taiwan \\ ${ }^{5}$ Department of Obstetrics and Gynecology, Chang Gung Memorial Hospital-Kaohsiung Medical Center, \\ Chang Gung University College of Medicine, Taipei, Taiwan
}

\begin{abstract}
The application of antioxidants in order to decrease injures of implanted cells has been an important issue for transplantation therapy. Coenzyme Q10 is widely distributed in animals and humans. It plays an important role in cellular energy production in the mitochondrial respiratory chain. Coenzyme Q10 has been demonstrated to have a potential role in the prevention and treatment of heart diseases by improving cellular bioenergetics. Mesechymal stem cells are a leading candidate for cell-based therapies. Researchers hypothesized that the condition of damaged myocardium could be improved with coenzyme Q10 supplementation therapy. Cell survival rate would be increased due in part to the decreased apoptosis and necrosis of cardiomyocytes in ischemic and reperfusion injures. In this study, a combination therapy of mesechymal stem cells transplantation and coenzyme Q10 supplementation for acute myocardial infarct showed promising results in vivo. Left ventricular function was notably improved with only small areas of fibrosis formation.
\end{abstract}

Key words: Cells therapy, acute myocardial infarction, Coenzyme Q10, mesenchymal stem cell, ischemic and reperfusion injures

\section{INTRODUCTION}

Cardiogenic shock is a state of inadequate tissue oxygen perfusion caused by cardiac diseases. Acute Myocardial Infarction (AMI) accounts for $5-10 \%$ of the incidence of cardiogenic shock (Cheng et al., 2009). Myocardial Infarction (MI) damages myocardiocytes through a sudden deprivation of circulating blood (Zhang et al., 2009a). Mesenchymal Stem Cells (MSCs) are multipotent stem cells found in the stroma of bone marrow (Baksh et al., 2004). MSCs can be induced to differentiate into a wide array of cells types including osteoblasts, chondrocytes, myocytes, adipocytes and neuronal cells (Mathieu et al., 2009). MSCs can be maintained in culture for long periods without losing their multipotency. Recent research indicates that MSCs are immuno-priveleged making them ideal candidates for allogenic transplantation therapies. The cell marker CD271 is one of the most differentially expressed markers of MSCs (Quirici et al., 2002; Kuci et al., 2010). The CD271 (+) MSCs have been demonstrated the potential of differentiation. As few as 50,000 uncultured MSCs have been shown to promote bone repair in vitro. Stem cell therapy has shown great potential in the field of regenerative cardiovascular medicine. MSCs may be a leading candidate for cell-based therapies for the ischemic heart (Abarbanell et al., 2009). Coenzyme Q10 is a component of the electron transport chain in the mitochondria for ATP synthesis (Birnbaum et al., 1996; Molyneux et al., 2008). The reduced form of CoQ10 is also an antioxidant. It is the only endogenously synthesized lipophilic antioxidant (Neuzil et al., 1997; Khatta et al., 2000). Several experimental studies have shown that coenzyme Q10 reduces the depletion of ATP,

Corresponding Author: Yan-Der Hsuuw, Department of Biological Science and Technology, 
enhances the recovery of cardiac function after ischemic-reperfusion injures and reduces the incidence of reperfusion arrhythmias (Satoh et al., 1995). In translational medicine, porcine models can reflect clinical conditions in cases of AMI. Physiologically, pigs possess many baseline characteristics similar to those of human beings including heart rate, $\mathrm{LV}$ blood pressure and $\mathrm{LV}$ Ejection Fraction (LVEF).

Researchers isolated and purified specific CD271positive mesenchymal stem cells from pigs using a MACS MicroBead System. Researchers examined the improvement of left-ventricular function after acute myocardial infarct induction in response to a combination of Co-enzyme Q10 and purified CD271 positive MSCs transplantation. The results show that a combination Coenzyme Q10 and MSC transplantation therapy improve LV ejection fraction, myocardium fibrosis and angiogenesis.

\section{MATERIALS AND METHODS}

Experiment animals: All experimental animals received humane animal care in accordance with the guidelines for care and use of experimental animals. The animals used in this study were housed and fed unlimitedly in an animal shelter.

Bone marrow aspiration: Experimental animals were physically examined for body weight, eye condition, skin appearance and mucous CRT. Researchers used atropine, xylazine (Bayer, $2.2 \mathrm{mg} \mathrm{kg}^{-1}$ I.M) and Zoletil-50 (Virbac, $6 \mathrm{mg} \mathrm{kg}^{-1}$ I.M) for general anesthesia that was maintained with an inhalation of $1.5 \%$ isoflurane (Halocarbon) in the procedures. Researchers collected $20 \mathrm{~mL}$ of bone marrow aspirate from femurs using a bone marrow needle.

\section{Mesenchymal Stem Cell (MSC) isolation and culture:} Bone marrow $(20 \mathrm{~mL}$ ) was aspirated from the femur stifle of pigs under general anesthesia. Bone marrow was prepared as described through RBC lysis and Lymphoprep separation (Axis-shield). Cells were cultured in low-glucose Dulbecco's modified Eagle's medium (Invitrogen) with 10\% FBS and 1\% penicillin-streptomycin and incubated at $37^{\circ} \mathrm{C}$ in a humidified atmosphere with $5 \%$ $\mathrm{CO}_{2}$. After 5 days of culturing, non-adherent cells were removed and adherent cells were incubated with CD271-PE antibodies for $10 \mathrm{~min}$ followed by capture with anti-PE microbeads for $15 \mathrm{~min}$ at $4^{\circ} \mathrm{C}$. A MACS apparatus was used to collect the magnetically labeled cells which were then cultured in DMEM (Invitrogen) with 10\% MSC-qualified FBS (Invitrogen) and 1\% penicillinstreptomycin and incubated at $37^{\circ} \mathrm{C}$ in a humidified atmosphere with $5 \% \mathrm{CO}_{2}$.

After 7 days in culture, $4 \times 10^{4}$ cells were collected for myocardial transplantation; MSCs were identified by staining with an anti-CD271 antibody with FITC fluorescence (Fig. 1).

Model of acute myocardial infarction: Experimental animals were anesthetized with atropine, xylazine and Zoletil-50. Experimental animals were treated with
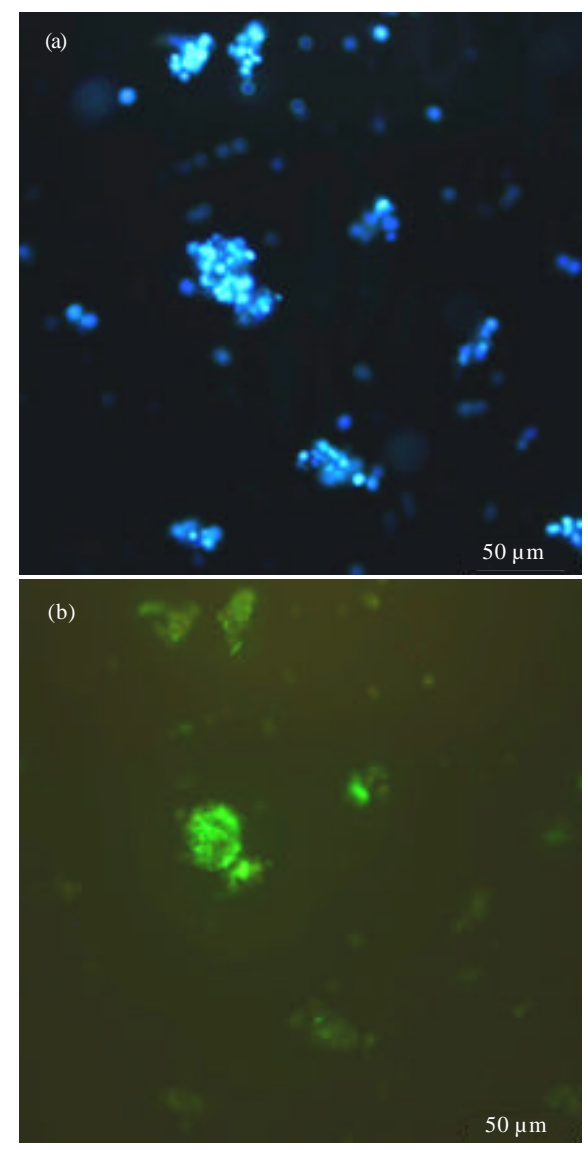

Fig. 1: Identification of mesenchymal Stem Cells (MSCs). Seed the CD271 (+) positive selected MSCs in $10 \mathrm{~cm}$ culture dish at a density of approximately $4 \times 10^{4}$ cells/dish in low-glucose DMEM (Invitrogen), the MSC attached on the dish surface and were stained by a) BIS and b) anti-CD271 antibody with FITC identify CD-271 (+) MSCs. The green fluorescence is approximately 95\% match with the BIS blue fluorescence 

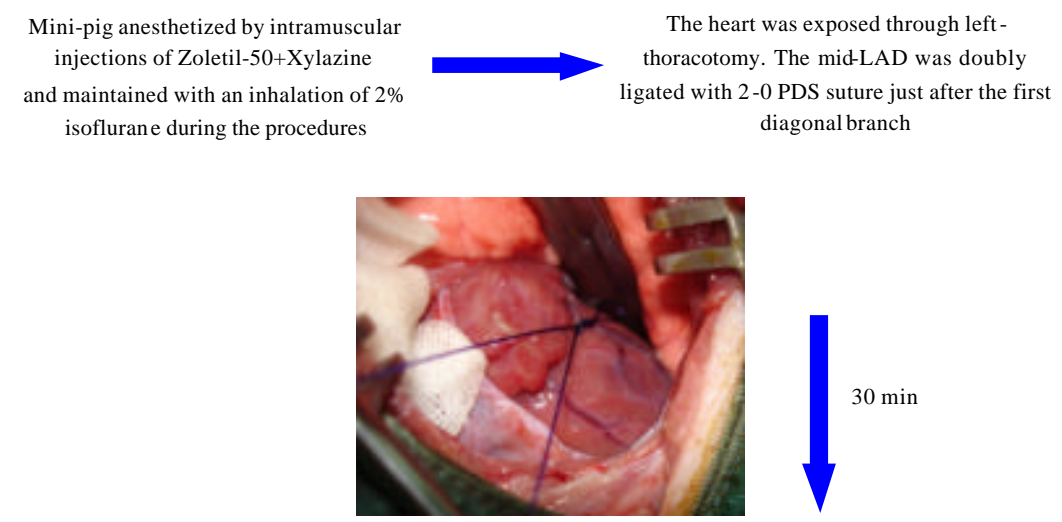

Myocardial infarction was confirmed with ECG
Observe a rapid whitish discoloration of

LV anterior chamber wall

Fig. 2: The procedures of AMI induction. The pigs were anesthetized with zoletil and xylazine before LAD ligation and maintained by inhalation of $2 \%$ isoflurane. The first branch of left coronary artery was ligated for $30 \mathrm{~min}$ to induce AMI. AMI was confirmed with the whitish appearance of myocardium and EKG S-T segment elevation

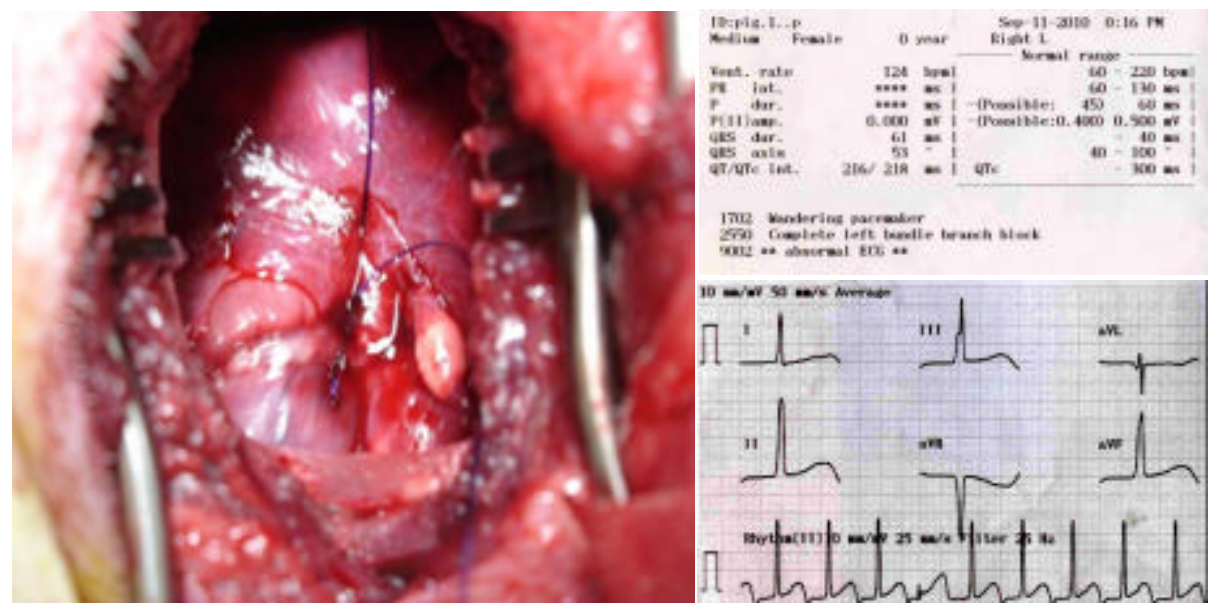

Fig. 3: EKG after AMI induction. The first branch of left coronary artery was ligated for $30 \mathrm{~min}$ to induce AMI. EKG S-T segment elevation was obvious after LAD first branch ligation. The condition was lasted for $30 \mathrm{~min}$. The appearance of myocardium (green arrow) was discolored gradually

endotracheal intubation and positive pressure ventilation with room air and oxygen (Fig. 2). An inhalation of $2 \%$ isoflurane was maintained during the procedures; an electrocardiogram monitor (Nihon Kohden Corportation) was connected to all four limbs and defibrillator was readied for use as needed. About $10 \mathrm{~mL}$ of blood was collected and an IV infusion was set up with Ringer's solution. Under sterile conditions, the heart was exposed through left-thoracotomy. An artificial MI was established through, double ligation of the mid-LAD for $30 \mathrm{~min}$ using a 2-0 PDS suture on the first diagonal branch. The regional myocardial infarct-ischemia area was confirmed by observing a rapid whitish discoloration of the anterior wall of the LV. Acute anterior wall myocardial infarction was also confirmed through observation of an S-T segment elevation of the ECG during the procedure (Fig. 3).

Cell delivery and Coenzyme Q10 supplementation: Intramyocardial or intracoronary injection of stem cells has been proposed to be a potential therapy for treating infarcted myocardium. In some models, animals implanted 
with stem cells post-MI have shown some favorable effects on cardiac remodeling and function. Researchers expected that MSCs would survive longer and better preserve cardiomyocytes in damaged myocardium. In the experiment, researchers delivered cultured MSCs into the myocardium of the infarct area using a $27 \mathrm{G}$ syringe after AMI induction immediately. Each pig in a CoQ10 experimental group was supplied with $60 \mathrm{mg}$ coenzyme Q10 (Rx Vitamin) after AMI.

Detection of apoptosis or necrosis: TUNEL assays and apoptotic nuclei: MSCs were treated with different concentrations of coenzyme Q10 $\left(1,2.5\right.$ and $\left.10 \mu \mathrm{g} \mathrm{mL}^{-1}\right)$ to study pathways of MSC death. TUNEL and nuclear staining of positive cells was examined in 3 randomly chosen HPFs.

\section{Functional assessment by echocardiography:}

Transthoracic echocardiography was performed preoperatively and on day 7 after AMI induction while under anesthesia as described previously using a commercially available Echocardiographic System (UF-750XT) that was equipped with two 3.5 and $5.0 \mathrm{MHZ}$ scanner-array transducers for animals (Fukuda Denshi Co., Hongo, Bunkyo-Ku, Tokyo, Japan) (Fig. 4). The animals were placed in a right recumbency position and left ventricular internal dimensions (i.e., End-Systolic Diameter (ESD) and End-Diastolic Diameter (EDD)) were measured. The LVEF was calculated as:

$$
\operatorname{LVEF}(\%)=\frac{(\text { LVEDD-LVEDS })}{(\text { LVEDD })} \times 100
$$

Where:

LEVDD $=($ LV End-Diastolic Diameter $)$

LVEDS $=($ LV End-Diastolic Stiffness)

All measurements were performed by an animal cardiologist blinded to the identity of the treatment and non-treatment groups.

New vessel density in infarct area: Gross hearts were sectioned into seven pieces at $1 \mathrm{~cm}$ intervals after animal sacrifice. These samples were examined by the laboratory of pathology. The sections were processed with hematoxylin and eosin. Pathology results were obtained for the infarct, junctional and non-infarction areas. Three sections of the infarct area were chosen for each pig. Randomly selected High-Power Fields (HPF) were analyzed for each section. New arterioles $(\leq 100 \mu \mathrm{m}$ in diameter) were examined and the mean number for each animal was determined by summation.
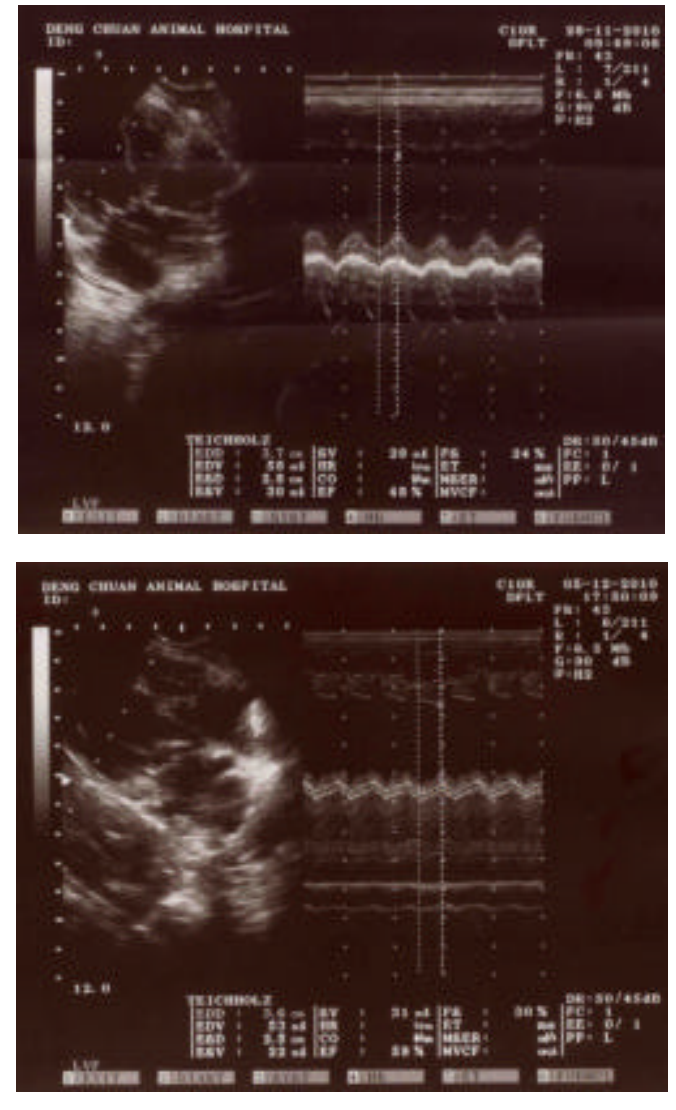

Fig. 4: Echocardiography measure of left ventricular function. The LV ejection fraction and fraction shortening was measured by echocardiography before and after $\mathrm{MI}$ induction

\section{RESULTS AND DISCUSSION}

Results of MSC graft on AMI: Experimental animals were equally divided into four groups: A) AMI alone: B) AMI plus CoQ10 supplementation: C) MSCs were implanted into the junction of infract-ischemia area after AMI induction: D) MSCs were injected into the junction of infract-ischemia area and provided CoQ10 supplementation. The experimental animals were sacrificed on day 7 after AMI induction. Researchers chose three fields in every section so there were nine chosen fields in each group to compare the area of fibrosis. The AMI plus MSC with CoQ10 supplement group showed the best fibrosis inhibition and preservation of the arrangement of the myocardium. Combination therapy of MSCs and CoQ10 showed the best results. MSCs alone or CoQ10 supplement groups showed partial improvement but the infarct area and fibrosis conditions were larger compared to the MSC plus CoQ10 group. 
Estimation of LV function with MSC graft therapy for AMI was examined by echocardiography before the animals were sacrificed. The results showed that the ventricular ejection fraction and fraction shortening were obviously improved by MSC grafting alone or MSC grafting combined with CoQ10 supplementation (Fig. 5). The areas of myocardium damage were smaller and the myocardial structure remained more intact in MSC implanted groups. MSC implantation combined with CoQ10 supplementation showed the smallest fibrosis area and the arrangement of the myocardium was preserved. These results show a great improvement in the Porcine Model and potentially promising effects of MSC implantation plus CoQ10 therapy for AMI (Fig. 6).

Vascular density in infracted myocardium and epicardium after treatment with stem cells and Coenzyme Q10: Angiogenesis in the infracted myocardium was examined as described in the study, the test group
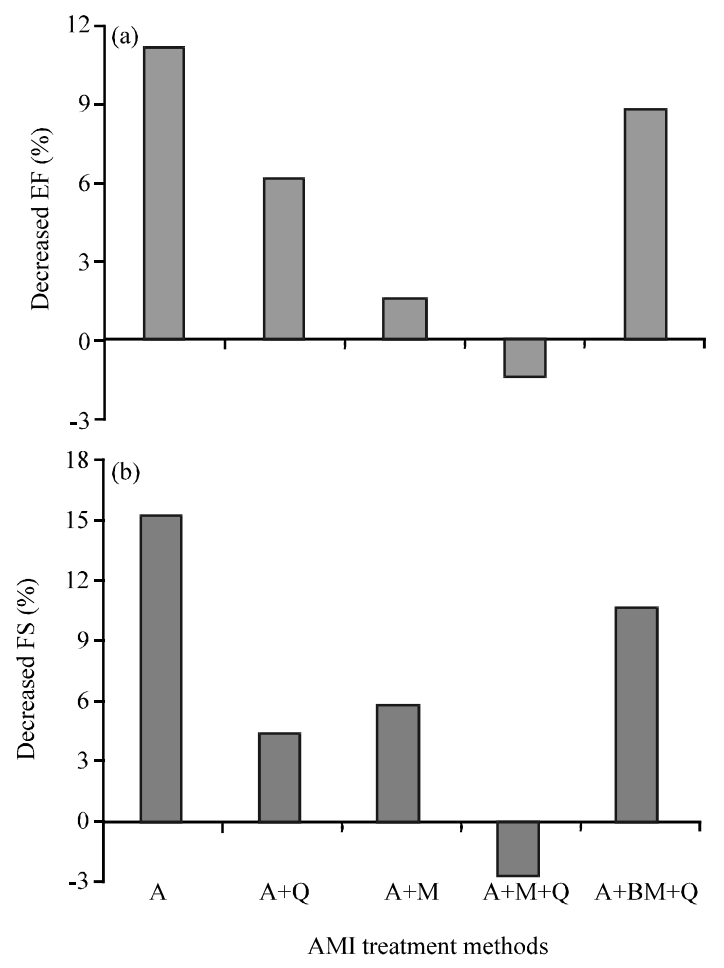

Fig. 5: Effects of MSC implantation on AMI. Ventricular ejection fraction and fraction shortening values were measured in M-mode echocardiography after MSC implantation therapy. A: acute myocardial infarct alone, Q: Coenzyme Q10 supplement, M: mesenchymal stem cell, BM: Un-purified bone marrow stromal cell. AMI + MSC + CoQ10 group showed the best improvement of EF and FS measurement. a) EF difference in AMI treatment; b) FS diffence in AMI treatment subjects were evaluated using histopathological assay. The micro-vessel density in the CoQ10 plus MSC group was significantly increased in comparison with AMI alone, AMI plus CoQ10 and AMI plus MSC implant (Fig. 7).

This study demonstrates that LV functions after AMI may benefit from a combination therapy of

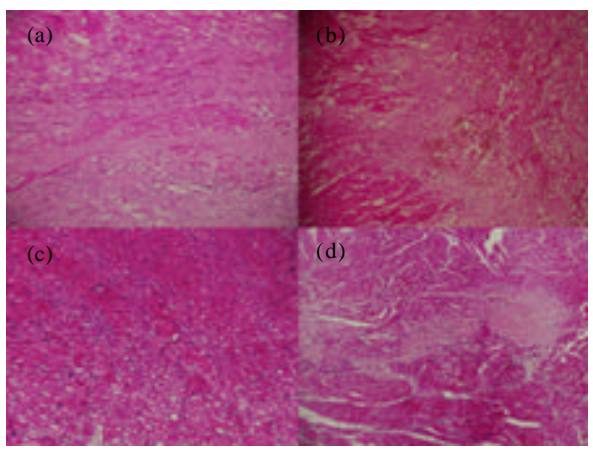

Fig. 6: The histopathologic examination after AMI damage and treatment. The diffuse fibrosis was noticed and no regeneration in a) AMI control group. The necrosis area decreased obviously in b) CoQ10 supplement group. The myocardium damage was less and myocardial appearance remains in c) $\mathrm{AMI}+\mathrm{MSC}$ group. The AMI + MSC + CoQ10 group showed the least fibrosis area and d) arrangement of myocardiocytes perseverated

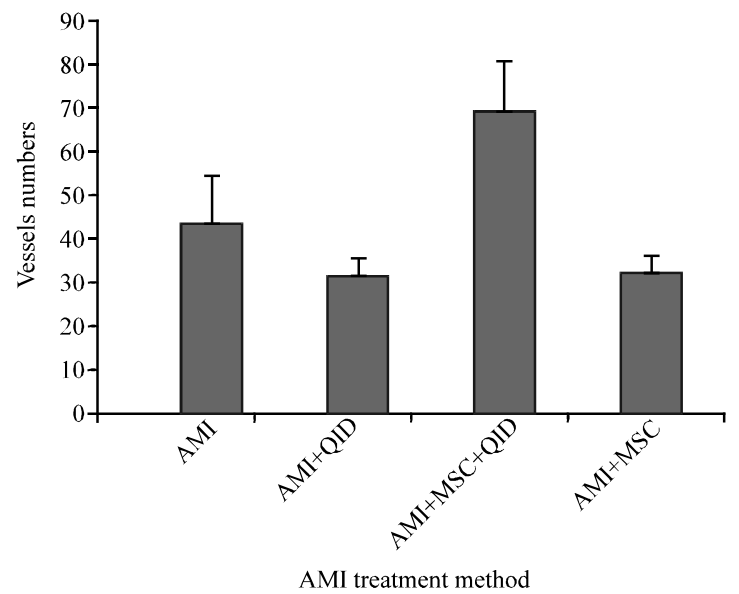

Fig. 7: Identification of small vessel $(\leq 100 \mu \mathrm{m})$ distribution on myocardium. Using $\mathrm{H} \& \mathrm{E}$ staining (on day 7 following AMI induction). The number of arterioles in infarct area of LV myocardium was substantially higher in AMI + MSC + Q10 group than other groups on day 7 following stem cells implantation 
MSC implantation with CoQ10 supplementation. CoQ10 supplementation increased the overall area of surviving myocardium by improving the microenvironment of infarct area. CoQ10 may help to improve nitric oxide bioactivity by scavenging any generated superoxide species as well as free radicals. A greater rate of conversion of nitric oxide to preoxynitite by superoxide anion can help to protect against myocardial nitrosative damages. In the histopathology assays, myocardial injury was less and myocardial structure remained. These results demonstrate the potential of COQ10 application in the treatment of cardiac disorders. A combination therapy of MSC implantation with CoQ10 supplementation showed the smallest areas of fibrosis. An initial trial of a recommended CoQ10 concentration for MSCs in culture was beneficial. Improvement of the myocardial infarct environment by CoQ10 in combination with the paracrine effects of MSCs promoted angiogenesis and prevented the further apoptosis of cardiomyocytes, leading to the preservation of cardiac function and structure. MSCs release VEGF and basic fibroblast growth factor and thereby exert beneficial effects on ischemia/reperfusion damages. Apart from the paracrine effects of MSCs on neighboring cells, it is also possible that injected MSCs served as chemoattractors and feeders for circulating hematopoietic stem cells and thereby further promoted resolution of injury.

The most frequent cause of acute $\mathrm{MI}$ is associated with an accumulation of myocardial plaque that occurs over a period of years or decades. Although, the prognosis of patients with cardiogenic shock has improved over time because of aggressive reperfusion strategies, the in hospital mortality rate from cardiogenic shock remains high (Akar et al., 2009). MSC therapy perhaps represents a new opportunity to enhance cardiac performance through a trans-differentiation of injected stem cells into myocardium to replace or repair damaged tissues or cells (Choi et al., 2009). There is accumulating experimental evidence for the application of cellular transplantation as a strategy to improve myocardial function and reduce scar burden due to MI (Amado et al., 2006; Choi et al., 2009). MSCs can differentiate into different tissues originating from mesoderm. Other studies of MSCs have used different methods of isolation and expansion and have identified MSCs through a different characterization method. The Low-Affinity Nerve Growth Factor Receptor (LNGFR), now clustered as CD271 was the most differentially expressed marker on MSCs. CD271 has served as a preferred marker for the purification of homogeneous populations of cells that contain BM and MSC activity such cells secrete a variety of cardioprotective or angiogenic cytokines in response to Ischemia-Reperfusion injury (I-R). In addition, MSCs engraft into injured myocardium and have the potential to differentiate into myocardiaocytes. Autologous transplantation of mesenchymal cells has been clinically applied to treat patients with ischemic heart diseases using catheter-based approaches that inject cells directly into the ischemic myocardium ( $\mathrm{Xu}$ et al., 2010). This approach has shown some favorable effects on cardiac remodeling and function (Zhang et al., 2009b). Clinical trials have shown that $\mathrm{CoQ} 10$ pretreatment enhances preservation of the myocardium during coronary artery bypass surgery. CoQ10 reduces myocardial ischemia-reperfusion injury induced by oxidative stress by suppressing the formation of reactive oxygen species (Maulik et al., 2000). A reduction of oxidative stress in conjunction with an increased antioxidant reserve in CoQ10-fed hearts suggests that CoQ10 functions by improving the antioxidant reserves of the heart. Oral COQ10 supplementation improves functional capacity, endothelial function and LV contractility in CHF without any side effects (Belardinelli et al., 2006). Exogenously administered CoQ10 nonspecifically incorporates into cell membranes and into various subcellular fractions and organelles such as mitochondrial membranes and sarcoplasmic reticular membranes (Czyz and Wobus, 2001). A significant number of reports in the literature support a role for oxygen free radicals and oxidative stress in ischemia-reperfusion injury (Tenney and Discher, 2009; Zhang et al., 2009b).

\section{CONCLUSION}

The combination therapy of mesenchymal stem cells transplantation and coenzyme Q10 supplementation for acute myocardial infarct showed promising results. Left ventricular function was notably improved with only small areas of fibrosis formation. Further studies shall be considered to confirm the benefit outcomes.

\section{ACKNOWLEDGEMENTS}

Researchers would like to thank Prof. Dr. Liang-Chou Hsia and Dr. Jai-Wei Lee of National Ping-Tung University of Science and Technology for help in preparation of this manuscript.

\section{REFERENCES}

Abarbanell, A.M., A.C. Coffey, J.W. Fehrenbacher, D.J. Beckman, J.L. Herrmann, B. Weil and D.R. Meldrum, 2009. Proinflammatory cytokine effects on mesenchymal stem cell therapy for the ischemic heart. Ann. Thorac. Surg., 88: 1036-1043. 
Akar, A.R., S. Durdu, M. Arat, M. Kilickap and N.O. Kucuk et al., 2009. Five-year follow-up after transepicardial implantation of autologous bone marrow mononuclear cells to ungraftable coronary territories for patients with ischaemic cardiomyopathy. Eur. J. Cardiothorac Surg., 36: 633-643.

Amado, L.C., K.H. Schuleri, A.P. Saliaris, A.J. Boyle and R. Helm et al., 2006. Multimodality noninvasive imaging demonstrates in vivo cardiac regeneration after mesenchymal stem cell therapy. J. Am. Coll. Cardiol. 48: 2116-2124.

Baksh, D., L. Song and R.S. Tuan, 2004. Adult mesenchymal stem cells: Characterization, differentiation and application in cell and gene therapy. J. Cell. Mol. Med., 8: 301-316.

Belardinelli, R., A. Mucaj, F. Lacalaprice, M. Solenghi and G. Seddaiu et al., 2006. Coenzyme Q10 and exercise training in chronic heart failure. Eur. Heart J., 27: 2675-2681.

Birnbaum, Y., S.L. Hale and R.A. Kloner, 1996. The effect of coenzyme Q10 on infarct size in a rabbit model of ischemia/reperfusion. Cardiovasc Res., 32: 861-868.

Cheng, J.M., S.D.A. Valk, C.A. den Uil, M. Van Der Ent and W.K. Lagrand et al., 2009. Usefulness of intra-aortic balloon pump counterpulsation in patients with cardiogenic shock from acute myocardial infarction. Am. J. Cardiol., 104: 327-332.

Choi, D., K.C. Hwang, K.Y. Lee and Y.H. Kim, 2009. Ischemic heart diseases: current treatments and future. J. Cont. Release, 140: 194-202.

Czyz, J. and A. Wobus, 2001. Embryonic stem cell differentiation: the role of extracellular factors. Differentiation, 68: 167-174.

Khatta, M., B.S. Alexander, C.M. Krichten, M.L. Fisher, R. Freudenberger, S.W. Robinson and S.S. Gottlieb, 2000. The effect of coenzyme Q10 in patients with congestive heart failure. Ann. Intern. Med., 132: 636-640.

Kuci, S., Z. Kuci, H. Kreyenberg, E. Deak and K. Putsch et al., 2010. CD271 antigen defines a subset of multipotent stromal cells with immunosuppressive and lymphohematopoietic engraftment-promoting properties. Haematologica, 95: 651-659.

Mathieu, M., J. Bartunek, B. El Oumeiri, K. Touihri and I. Hadad et al., 2009. Cell therapy with autologous bone marrow mononuclear stem cells is associated with superior cardiac recovery compared with use of nonmodified mesenchymal stem cells in a canine model of chronic myocardial infarction. J. Thorac. Cardiovasc Surg., 138: 646-646.
Maulik, N., T. Yoshida, R.M. Engelman, D. Bagchi, H. Otani and D.K. Das, 2000. Dietary coenzyme Q (10) supplement renders swine hearts resistant to ischemia-reperfusion injury. Am. J. Physiol Heart Circ Physiol., 278: H1 084-H1090.

Molyneux, S.L., J.M. Young, C.M. Florkowski, M. Lever and P.M. George, 2008. Coenzyme Q10: Is there a clinical role and a case for measurement. Clin. Biochem. Rev., 29: 71-82.

Neuzil, J., P.K. Witting and R. Stocker, 1997. Alphatocopheryl hydroquinone is an efficient multifunctional inhibitor of radical-initiated oxidation of low density lipoprotein lipids. Proc. National Acad. Sci., 94: 7885-7890.

Quirici, N., D. Soligo, P. Bossolasco, F. Servida, C. Lumini and G.L. Deliliers, 2002. Isolation of bone marrow mesenchymal stem cells by anti-nerve growth factor receptor antibodies. Exp. Hematol., 30: 783-791.

Satoh, K., A. Yamato, T. Nakai, K. Hoshi and K. Ichihara, 1995. Effects of 3-hydroxy-3-methylglutaryl coenzyme A reductase inhibitors on mitochondrial respiration in ischaemic dog hearts. $\mathrm{Br}$. J. Pharmacol., 116: 1894-1898.

Tenney, R.M. and D.E. Discher, 2009. Stem cells, microenvironment mechanics and growth factor activation. Curr. Opin. Cell Biol., 21: $630-635$.

Xu, Y.L., Y.H. Gao, Z. Liu, K.B. Tan and X. Hua et al., 2010. Myocardium-targeted ransplantation of mesenchymal stem cells by diagnostic ultrasoundmediated microbubble destruction improves cardiac function in myocardial infarction of New Zealand rabbits. Int. J. Cardiol., 138: 182-195.

Zhang, S.N., A. Sun, D. Xu, K. Yao and Z. Huang et al., 2009b. Impact of timing on efficacy and safetyof intracoronary autologous bone marrow stem cells transplantation in acute myocardial infarction: A pooled subgroup analysis of randomized controlled trials. Clin. Cardiol., 2: $458-466$.

Zhang, S.N., A.J. Sun, J.B. Ge, K. Yao, Z.Y. Huang, K.Q. Wang and Y.Z. Zou, 2009a. Intracoronary autologous bone marrow stem cells transfer for patients with acute myocardial infarction: A meta-analysis of randomised controlled trials. Int. J. Cardiol., 136: 178-185. 